\title{
Sustained Operations in Confined-Space Military Vehicles
}

\author{
Nico J. Delleman \\ TNO Human Factors, Soesterberg, The Netherlands \\ Paris Descartes University, Paris, France \\ Véronique Colaciuri \\ Emeric Wiederkehr \\ Paris Descartes University, Paris, France
}

Pierre J.L. Valk

TNO Human Factors, Soesterberg, The Netherlands

This paper reports 2 baseline studies and 1 experiment performed in a confined-space military vehicle concerning the effects on fitness and performance of time in a sitting posture and workstation characteristics. On average physical fitness decreased by slightly more than 10\% per hour, the observation performance decreased by $30 \%$ per hour, and the technical performance (of gunners) showed a relatively small decrease, i.e., less than 5\% per hour. So-called active breaks (changing sitting into standing and walking) led to a significant reduction in the decrease of physical fitness, almost reducing it to zero. Furthermore, the level of confinement was shown to affect physical fitness.

\section{fitness performance active breaks}

\section{INTRODUCTION}

Military vehicles are often characterised by confined-space workstations. Operations in these vehicles require sustained, relatively static postures. It is our common understanding as ergonomists that lack of posture variation leads to a gradual decrease of the level of fitness or, in other words, an increase in the level of discomfort and fatigue, and a decrease in task performance (speed, accuracy, task completion). On top of that, restrictions of space and nonoptimal positioning of displays and controls may lead to awkward postures. To get a better insight into what to expect from its personnel working in vehicles, the Royal Netherlands Army (RNLA) asked TNO Human Factors to determine the effects of sustained operations in confined-space military vehicles on fitness and performance. The aim is to make dose-effect relationships available (a) for technical issues concerning design and redesign of workstations to be decided upon by procurers of vehicles and (b) for operational issues concerning work-rest schedules for crews to be decided upon by commanders.

International standards No. ISO 11226:2000 [1] and EN 1005-4:2005 [2] represent state-of-theart evaluation of body postures. The quantitative 
evaluation criteria in these documents include, amongst others, holding times for head inclination forwards, trunk inclination forwards and upper arm elevation, and criteria for evaluation of the posture of the upper arm with respect to the trunk, and neck flexion/ extension (head inclination with respect to trunk inclination). Holding times are given in terms of minutes, for a certain range of postures only, i.e., those deviating considerably from the neutral posture (e.g., an unsupported trunk inclination forwards between $20^{\circ}$ and $60^{\circ}$ ). For the neutral posture or for relatively small deviations from the neutral posture of the major body segments like the trunk, head and upper arm, no such temporal information is presented. Concerning posture variation standard No. ISO 11226:2000 [1] only contains general information, promoting more and shorter alternating work and rest periods. From a literature review by Mathiassen and Christmansson [3] it may be concluded that additional short breaks may reduce discomfort in monotonous work, not necessarily affecting task performance in a negative way. Furthermore, this review led the authors of the current paper to the conclusion that so-called active breaks (instead of non-active breaks, i.e., rest in physical terms) may have a positive effect on discomfort. In the case of neutral or close to neutral postures of the main segments, the opportunity for posture variation depends on the level of confinement. A literature search by the authors of the current paper disclosed no studies on the effect of the level of confinement on fitness and performance. Concerning the relationship between posture and task performance Drury and Paquet [4] wrote that there were a limited number of studies. Only for head and trunk postures was a clear relationship with visual inspection performance found. The goal of the current project is to study the effects on fitness and performance of the time in a sitting posture (duration in terms of hours, neutral or close to neutral postures of the main segment, and effects of additional short breaks and workrest schedules, for instance) and workstation characteristics (e.g., level of confinement).

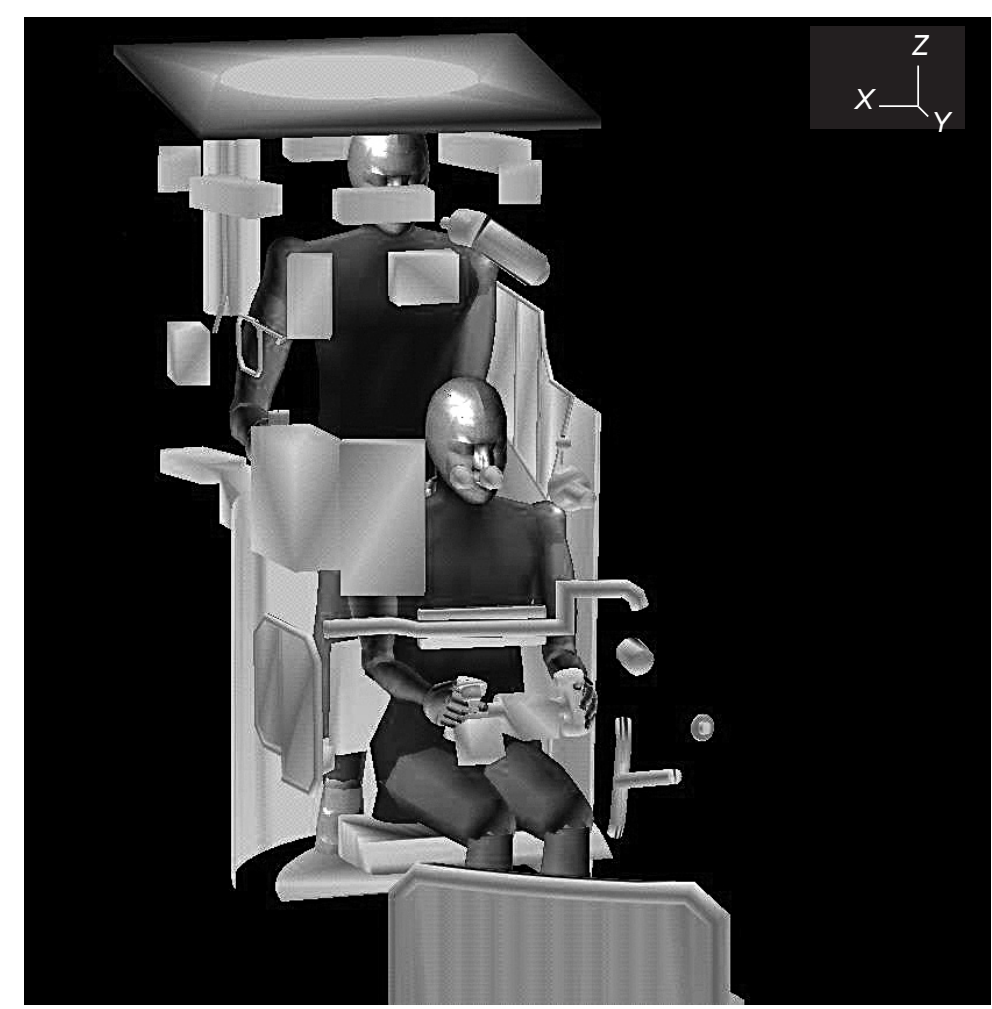

Figure 1. The gunner (lower position) and the commander (upper position) in their confined workstations in a Leopard 2-A5 MBT, visualised by digital human models in a computer-aided design (CAD) environment. Notes. The vehicle hull and other structural elements have been removed for reasons of clarity. 
At the start of the project various military RNLA vehicles were evaluated to select one as the object of the study. The Leopard 2-A5 Main Battle Tank (MBT) was chosen as it turned out to have the most confined workstations. Figure 1 shows a gunner (lower position) and a commander (upper position) as digital human models in a computer-aided design (CAD) visualisation of their workstations in the vehicle. So far in the project two baseline studies and one experiment have been done. They are described in sections 2-4 and followed by an overall discussion of the results in section 5 .

\section{BASELINE STUDY IN A SIMULATOR}

The goal of the first baseline study was to get insight into the effect of the duration of operation on gunners' fitness and technical performance. The study was performed during a pre-programmed military training activity. It should be mentioned that a gunner's working posture is not optimal, i.e., it is characterised by a slight forward inclination of the trunk and a slight extension of the neck to use the optics for scanning the outside world and aiming.

\subsection{Method}

Six male gunners participated in the study. Their age was 18-23 years, their stature 170-187 cm, and their body weight was $72-90 \mathrm{~kg}$. All received the standard education for becoming a gunner. At the time of the experiment 5 of them had worked $0.5-1.5$ years as a gunner, while one had just finished his educational program.

The gunners operated each in their own simulator, which was an exact copy of their workstation in a real Leopard 2-A5 MBT. In each of six sessions one of three test programs was offered to a gunner. The order of presenting the programs was balanced over the six sessions. A test program consisted of a certain panorama (landscape of grassland, heath, and some trees), disclosing various targets, generated by the computer and random in time and position.
The gunners needed on average $52.48 \mathrm{~min}$ (SD 5.46, range: 43-66) for each test program, in which 46 targets were presented. After that they were given a break of $17 \mathrm{~min}$ on average (SD 5.83, range: 7-26). The break was used for eating and drinking and for filling out rating scales (see end of this section). During the break they did not leave their seat in the workstation. The session after a break started at the same time for all gunners involved, i.e., when the subject who needed most time for the test program and the break activities had finished. The whole test lasted $\sim 6 \mathrm{hrs}$ and $40 \mathrm{~min}$.

The technical performance of the gunners comprised all prescribed preparatory actions as well as actual shooting performance. For each test program executed, the simulator produced a score, i.e., 100 minus a value based on the number of incorrect preparatory actions, and minus a value based on the number of targets missed, where a score of 70 was considered normal. The score obtained was used to analyse technical performance. The scores of subject 1 were considered unreliable by the military trainer involved, and therefore excluded from the data analysis. Subjects 2-6 indicated in one of the breaks that they would like to quit. They were encouraged to continue for at least one more session and all succeeded in doing that. However, the scores for these final sessions were not used due to the well-known phenomenon that subjects tend to finish with a final burst of energy leading to an enhancement of performance, which was not the subject of this study.

Immediately before and immediately after each test program the gunners were asked to rate their fitness in various ways. Three rating scales were used for this:

- Physical fitness. The gunner was asked to rate the extent to which he was physically fatigued by putting a cross on a visual analog scale with the terms none and extreme at the ends. Fitness was defined as the inverse of fatigue. The distance of the cross from both ends was measured and divided by the length of the scale, resulting in a score between 0 and $100 \%$ fitness. 
- Mental fitness. The gunner was asked to rate the extent to which he was mentally fatigued by putting a cross on a visual analog scale with the terms none and extreme at the ends. The same procedure for data processing was used as for the analysis of physical fitness.

- Localised postural discomfort. The gunner was asked to rate his postural discomfort in 40 regions shown on a diagram of the rear view of a human body (modified after Corlett and Bishop [5]), using a category-ratio scale by Borg [6] ranging from 0 (no discomfort) to 10 (extreme/maximal discomfort) [7].

\subsection{Results}

Figure 2 shows the decrease in the physical fitness of the gunners over time. Subjects were grouped according to their endurance, i.e., subject 6 , who had serious back complaints already at the start of the tests quitted after three sessions, subjects 2 and 3 finished after four sessions, and subjects 1 , 4 , and 5 completed all sessions. The fitness reduction rate was $28.1 \%$ per hour (beyond the first session) for subject $6,14.9 \%$ per hour for subjects 2 and 3, and 5.3\% per hour for subjects 1,4 , and 5 (similar rates for the 3 subjects). The average decrease in subjects $1-5$ over the first four sessions ( $4.5 \mathrm{hrs}$ ) was $10.7 \%$ per hour.

The results on mental fitness and localised postural discomfort resemble Figure 2. Postural discomfort appeared mainly in the neck, upper back, lower back, and buttocks.

Figure 3 shows that the technical performance of subject 6 decreased at the highest rate, i.e., $11.6 \%$ per hour. The performance of subjects 2 and 3 decreased by $4.1 \%$ per hour. Subjects 4 and 5 showed a reduction of $4.4 \%$ per hour, but only beyond $180 \mathrm{~min}$.

\section{BASELINE STUDY IN THE FIELD}

The goal of the second baseline study was to gain insight into the effect of the duration of an operation on the fitness of gunners and commanders, and on the observation performance of gunners. Furthermore, the effect of short active breaks (temporary changes in the sitting posture by standing and walking) on fitness was studied. The hypothesis was that active breaks lead to a lower rate of fitness decrease than nonactive breaks. The study was performed during a pre-programmed military training activity. As mentioned in section 2, gunners need a slight forward inclination of the trunk and a slight extension of the neck to use the optics for scanning the outside world and aiming. Commanders are able to operate in a reasonably neutral posture.

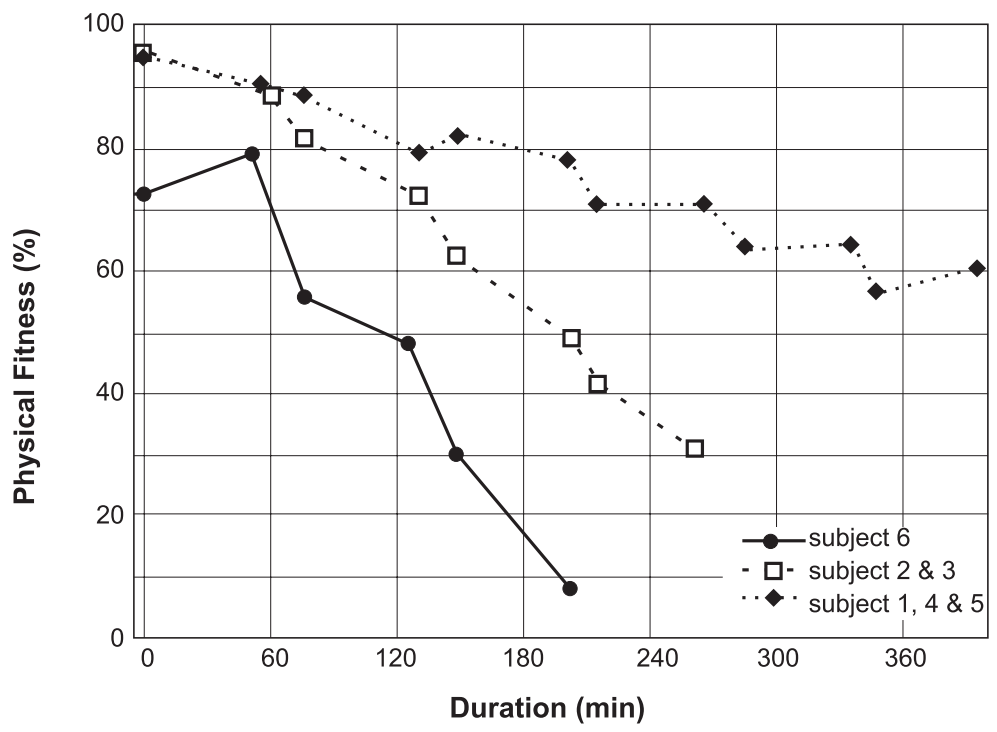

Figure 2. Baseline study in the simulator: gunners' physical fitness in time. 


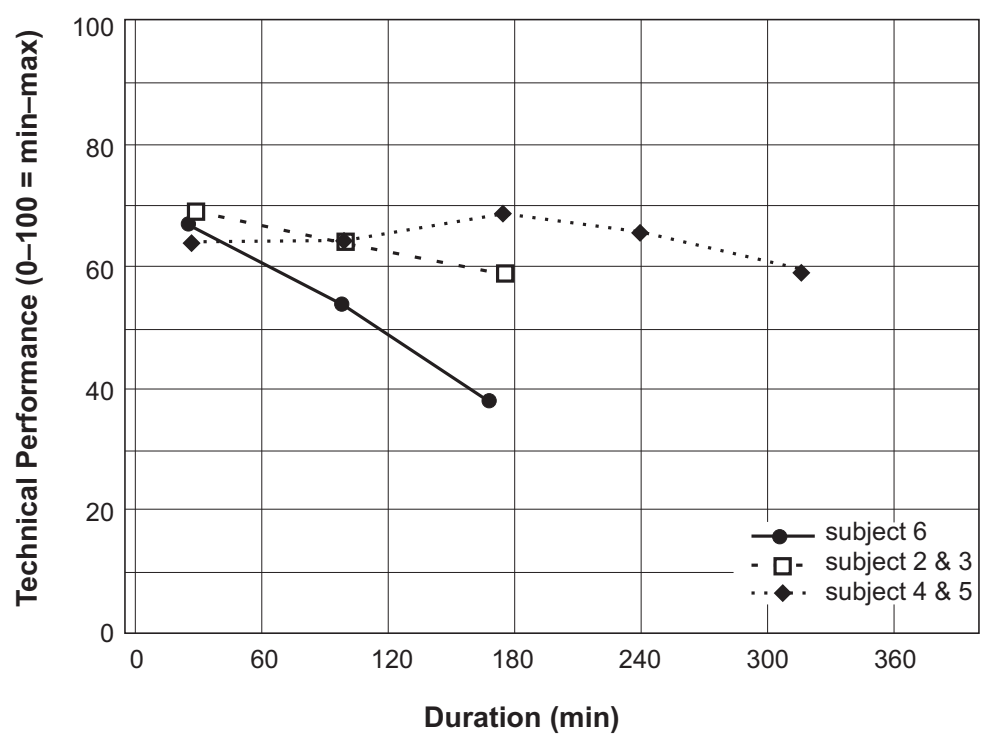

Figure 3. Baseline study in the simulator: gunners' technical performance in time.

\subsection{Method}

Four male gunners participated in the study. Their age was 18-25 years, their stature 176-195 cm, and their body weight was $75-90 \mathrm{~kg}$. All received the standard education for becoming a gunner. At the time of the experiment they had worked 1 week, 4 months, 4 months, and 2.5 years. Furthermore, 4 male commanders participated. Their age was 21-32 years, their stature 178$190 \mathrm{~cm}$, and their body weight was 72-95 kg. At the time of the experiment one had worked 8 months and the others 1 year.

Four Leopards were positioned at the edge of flat open grassland. A military trainer involved in the study could make various targets could appear in front of them, one at a time; they were remotely operated and random in time. The gunners had to scan the area for a target, as their field of view was not large enough to see the whole area at once. The procedure for testing was the same as the standard training procedure used. Each vehicle contained a gunner and a commander. Using a stopwatch the commander measured the time the gunner needed between "target up" and "tank" (target in sight). For each target the commanders were pre-warned through their head phones with the command "attention please" followed by the command "target up". The time was stopped when the gunner told the commander "tank". The gunners were not aware of any communication between the military trainer operating the targets and the commander in their vehicle. Targets that were not noticed by a gunner within the time given by the trainer were registered as missed.

The gunners and commanders operated in 25-min sessions, followed by 5-min breaks. In each session 13-15 targets were presented. A break was used for eating and drinking and for filling out rating scales. During all breaks the gunners did not leave their seats in the workstation. The commanders did not leave their workstation during the first four breaks (nonactive breaks), whereas they came out of the vehicle during the following four breaks (active breaks). The whole test lasted 4 hrs.

The observation performance of the gunners is defined as the time between "target up" and "tank". The results for the final session were not used, as a considerably higher number of targets was presented (24) by the military trainer as compared to the previous seven sessions.

Immediately before and immediately after each session (and once more at the end of the break after the final session) the gunners and the commanders were asked to rate their fitness in various ways. The same method as in the baseline study in the simulator (see section 2) was used. To get more insight into priorities for improvement of the working conditions of the 
gunners and commanders during regular daily operations, they were asked to rank eight items that were mentioned in several interviews with other gunners and commanders preceding the study (cf. Table 1).

TABLE 1. Baseline Study in the Field: Priorities for Improvement of Working Conditions According to Gunners and Commanders (Average Group Scores)

\begin{tabular}{lcc}
\hline Item & Gunn. & Comm. \\
\hline Quality of seat pan and backrest & 2.000 & 3.375 \\
Space available at workstation & 2.500 & 2.375 \\
Sustained seating & 3.000 & 5.125 \\
Temperature & 5.000 & 5.125 \\
Sustained task execution & 5.250 & 4.375 \\
Shocks and vibrations & 5.250 & 4.125 \\
Noise & 5.500 & 5.375 \\
Air pressure after a shot & 7.500 & 6.125 \\
\hline
\end{tabular}

Notes. Gunn.-gunners' ranking, Comm.-commanders' ranking; rank rating scale: 1-highest priority, 8-lowest priority.

The differences between the period with nonactive breaks (0-145 $\mathrm{min}$ ) and the period with active breaks (145-240 $\mathrm{min}$ ) for commanders as regards all fitness variables were tested with a Student $T$ test on the slopes of the linear regression lines fitted to the data points. These analyses were done for the gunners as well, i.e., the differences between their two periods of nonactive breaks (0-145 and 145-240 min) were analysed. The selected level of significance in all tests was $p=.05$ (one-tailed).

\subsection{Results}

Figures 4-5 show the decrease in physical fitness of the gunners and commanders over time. Up to 145 min (non-active breaks) the fitness reduction rate was $10.3 \%$ per hour for the gunners and $10.9 \%$ per hour for the commanders. For the gunners there was no significant change beyond $145 \mathrm{~min}$ (non-active breaks), while the active breaks for the commanders led to a significant reduction in the decrease of physical fitness.

The results on localised postural discomfort resemble Figures 4-5. Postural discomfort for the gunners appeared mainly in the neck, upper back, lower back, and buttocks. Postural discomfort for the commanders appeared mainly in the lower back, buttocks, and upper legs (back side).

Figures 6-7 show the decrease in mental fitness of the gunners and commanders over time. Up to $145 \mathrm{~min}$ (non-active breaks) the fitness reduction rate was $12.6 \%$ per hour for the gunners, and $6.5 \%$ per hour for the commanders. For the

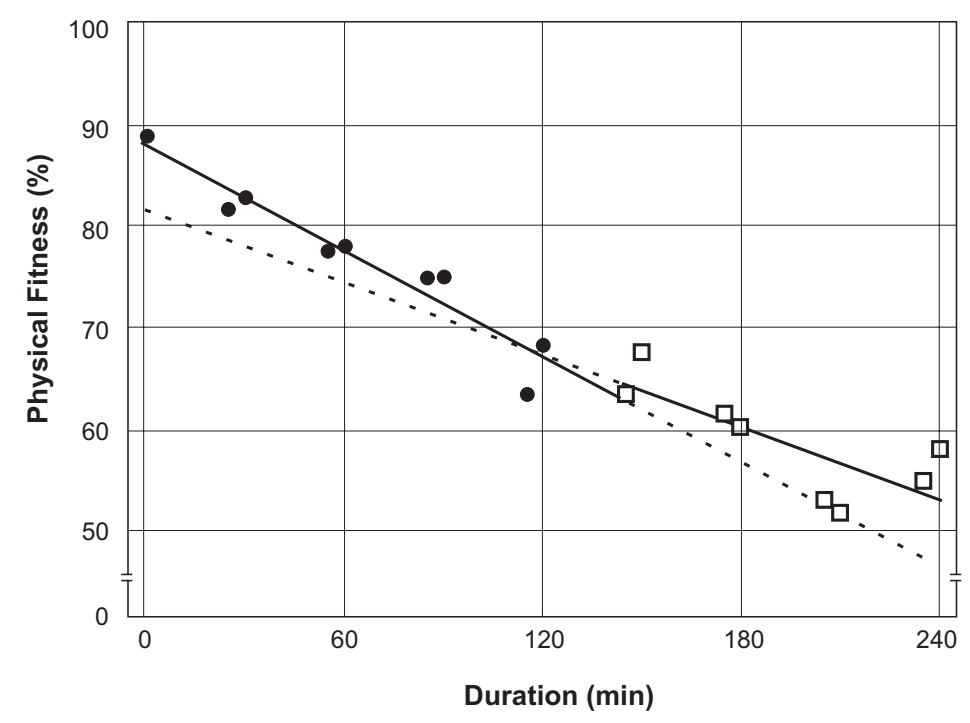

Figure 4. Baseline study in the field: gunners' physical fitness in time (average group data). Notes. Filled circles represent data points obtained in the period with non-active breaks for the commanders (0-145 min). Open squares represent data points obtained in the period with active breaks for the commanders (145$240 \mathrm{~min}$ ). For both periods the regression lines fitted to the data points are shown. 


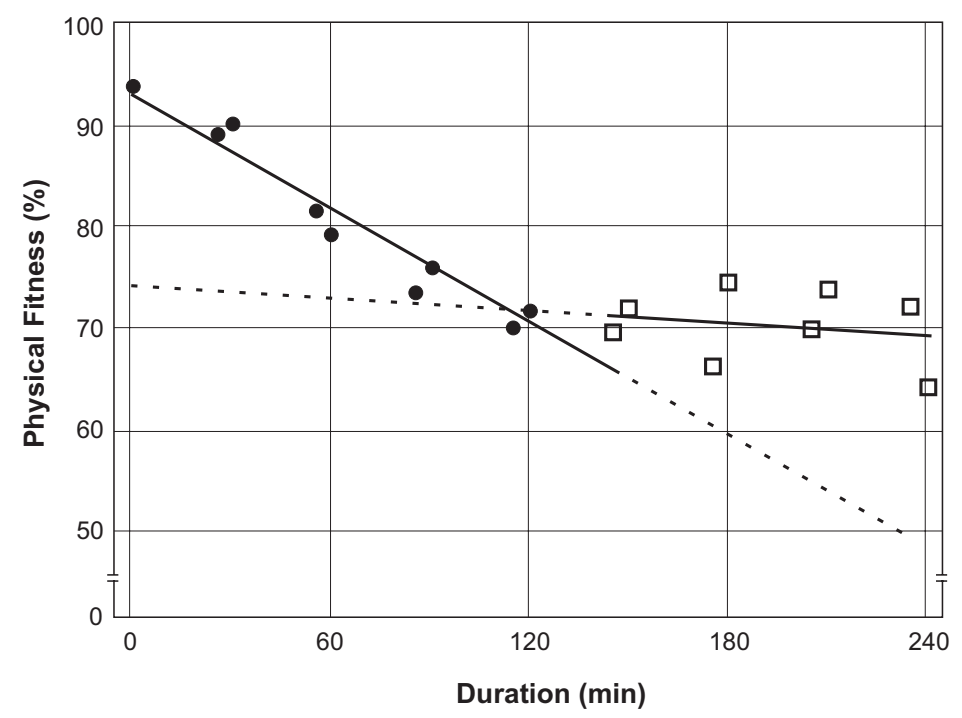

Figure 5. Baseline study in the field: commanders' physical fitness in time (average group data). Notes. Filled circles represent data points obtained in the period with non-active breaks (0-145 $\mathrm{min})$. Open squares represent data points obtained in the period with active breaks (145-240 min). For both periods the regression lines fitted to the data points are shown.

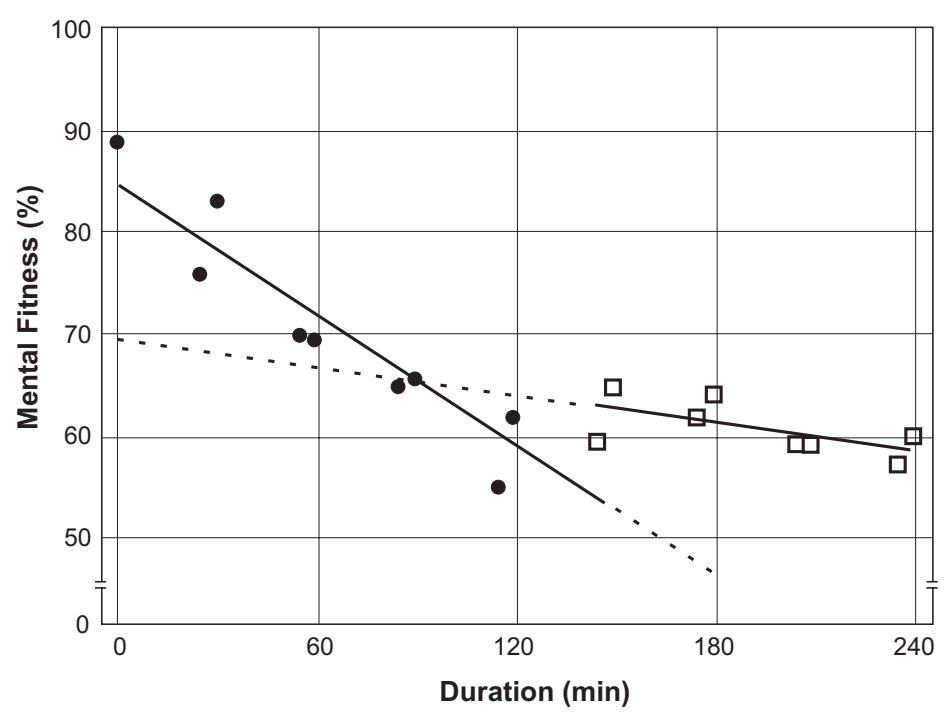

Figure 6. Baseline study in the field: gunners' mental fitness in time (average group data). Notes. Filled circles represent data points obtained in the period with non-active breaks for the commanders (0-145 min). Open squares represent data points obtained in the period with active breaks for the commanders (145$240 \mathrm{~min}$ ). For both periods the regression lines fitted to the data points are shown.

commanders there was no significant change beyond $145 \mathrm{~min}$ (active breaks), while for the gunners there was a significant reduction in the decrease of mental fitness.

Figure 8 shows the observation performance of the gunners. On average the performance decreased by $29.8 \%$ per hour. The number of targets missed was stable across the sessions (on average 1.1 target per session was missed by the gunners).

Table 1 contains the priorities for improvement of working conditions according to the gunners and the commanders. 


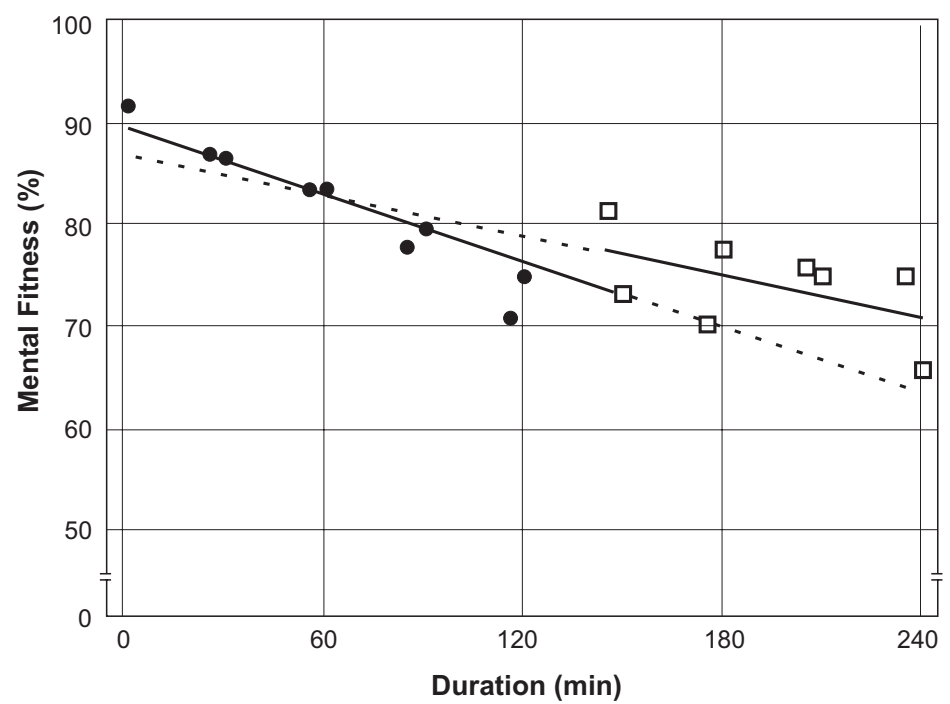

Figure 7. Baseline study in the field: commanders' mental fitness in time (average group data). Notes. Filled circles represent data points obtained in the period with non-active breaks (0-145 $\mathrm{min}$ ). Open squares represent data points obtained in the period with active breaks (145-240 $\mathrm{min})$. For both periods the regression lines fitted to the data points are shown.

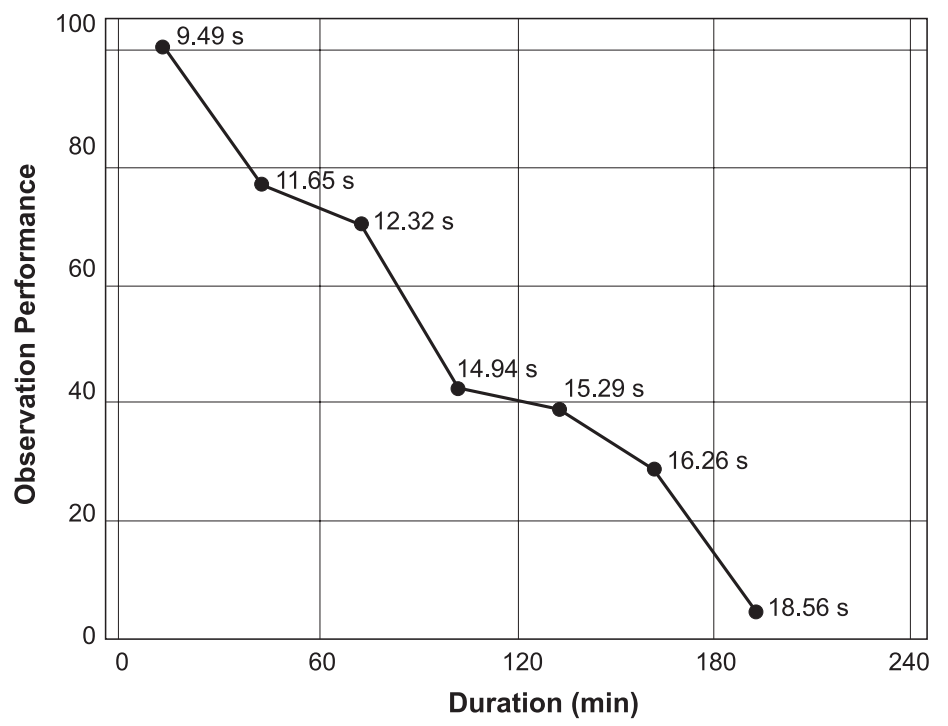

Figure 8. Baseline study in the field: observation performance (in seconds) of the gunners in time (average group data). Notes. Performance in the first session is given a reference score of 100 . Performance in other sessions was defined as $100-(($ observation time -9.49$) / 9.49) \cdot 100$.

\section{EXPERIMENTAL STUDY IN THE LABORATORY}

The goal of the experiment was to establish the effect of the level of confinement of the body on fitness and performance. The main hypothesis to be tested is that more confinement leads to lower fitness and performance.

\subsection{Method}

Five male and four female students participated in the experiment. Their age was 21-34 years, their stature $165-199 \mathrm{~cm}$, and their body weight 53-102 kg.

The experimental workstation consisted of a computer screen, a joystick, a normal office seat with a high backrest and without armrests, one fixed plate behind the backrest, and five 
adjustable plates for creating a certain level of confinement for the test subjects (Figures 9-10). The position of the plate in front of the knees determined the space for extending the knees and/ or leaning the trunk backwards. The positions of two upper leg plates and two upper arm plates determined the space for lateral posture variation of the trunk. The screen and the joystick were positioned and oriented in such a way that discomfort of the neck and right shoulder/upper arm would be minimal.

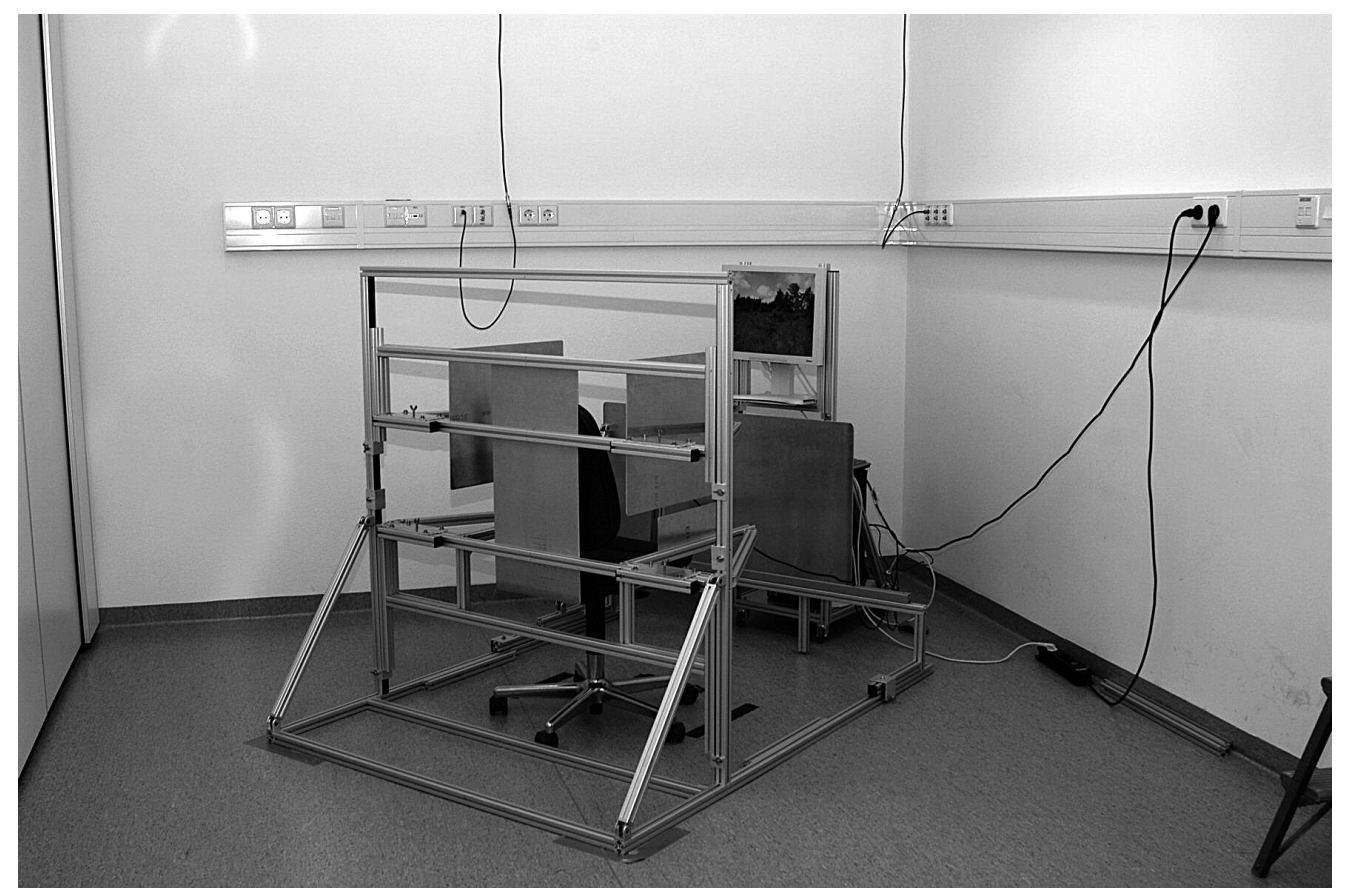

Figure 9. Experimental study in the laboratory: experimental workstation (rear view).

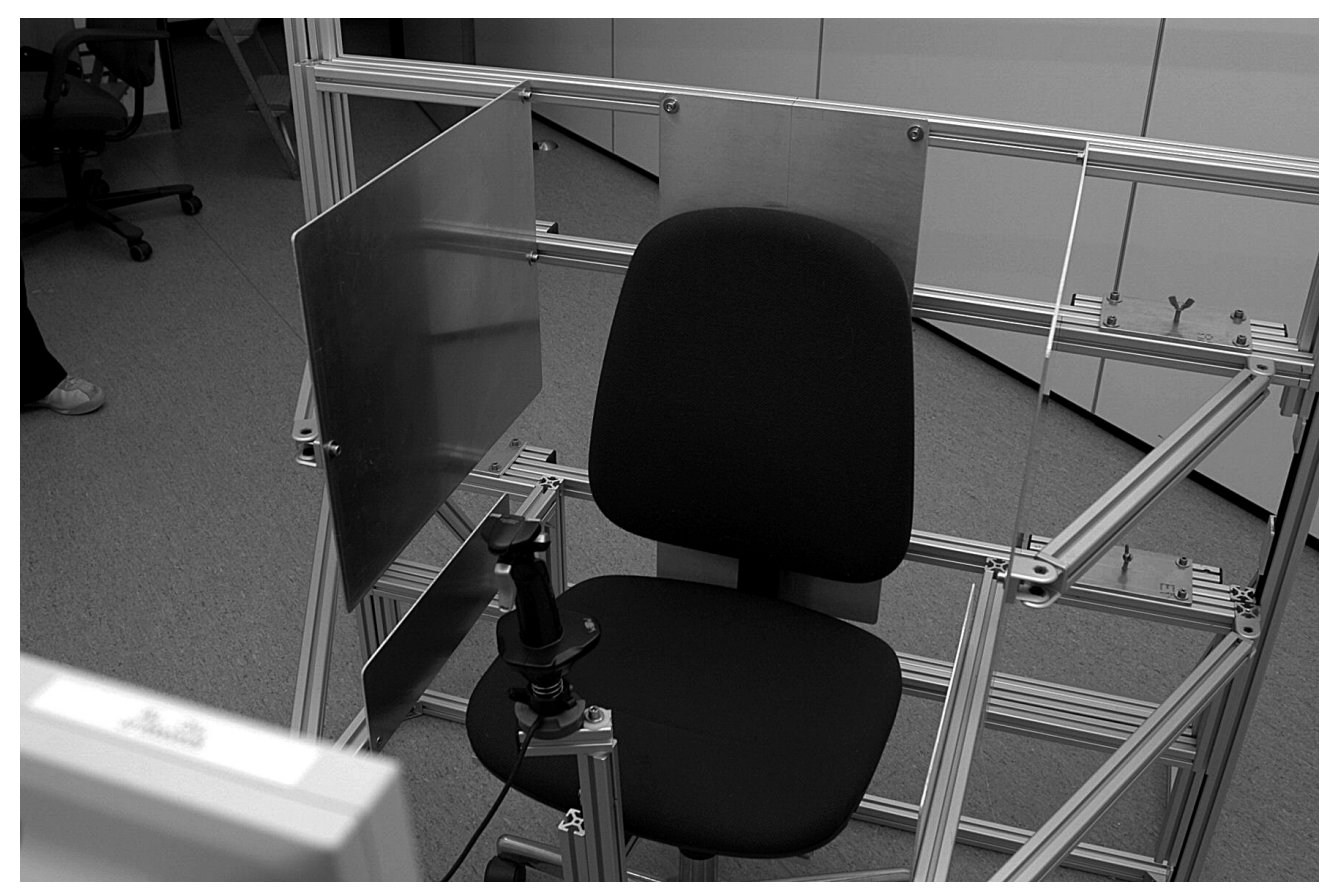

Figure 10. Experimental study in the laboratory: experimental workstation (front view). 
The experimental task consisted of finding a target (a contour of a tank) as quickly as possible by scanning a panorama (landscape of grassland, heath, and some trees), analogously to the procedure used in the baseline study in the field (see section 3). For this, the subjects operated a joystick with their right hand. Targets appeared computer-generated, random in time (denoted $\mathrm{T} 0$ ) and position in the panorama (T1 denoted the moment that the target entered the field-of-view). As soon as the subjects saw a target they had to press the "found" button on the joystick (T2). Then, they had to get the target within gun sight, and eliminate it by pressing the "fire" button on the joystick as quickly as possible (T3). After appearance of the target in the panorama, the subjects had $30 \mathrm{~s}$ to find and eliminate it.

Three experimental conditions were tested on three consecutive days, either three mornings or three afternoons:

- free: subject sitting in any freely chosen posture, i.e., without the plates confining the space for posture variation;

- fully confined: subject sitting upright, upper legs horizontal, and lower legs vertical, with all plates as close as possible to the body;

- fully confined $+15 \%$ for/aft space: same as the fully confined condition but having positioned the plate in front of the knees further away, i.e., increasing the for/aft distance between the patella front edge and the most prominent point on the backrest centreline by $15 \%$.

The order of presentation of the experimental conditions was balanced as much as possible.

The subjects operated in five 25-min sessions, each followed by a 5-min break. In each session 25 targets were presented. A break was used for eating and drinking and for filling out rating scales. During all breaks the subjects did not leave their seat. The whole test lasted $2.5 \mathrm{hrs}$.

The subjects' performance was defined by the following dependent variables (representing the time differences between distinct moments in time, as defined earlier in this section): T0-T1, T0-T2, T0-T3, T1-T2, T1-T3, and T2-T3. Targets which were not eliminated within $30 \mathrm{~s}$ after appearance in the panorama were registered as missed targets. The number of missed targets was used as a dependent variable.

Immediately before and immediately after each session (and once more at the end of the break after the final session) the subjects were asked to rate their fitness in various ways. The same method as in the baseline studies (see sections 2-3) was used. In addition, the subjects were asked to rate eye discomfort and headache, using the same scale as for localised postural discomfort. The results of all individual rating scales described were used as dependent variables. Concerning localised postural discomfort, the following dependent variables were constructed by grouping the 40 body regions into larger functional units: right shoulder-arm, left shoulder-arm, neck-back, and buttocks-legs.

The main effects of the experimental conditions (free, fully confined, and fully confined $+15 \%$ for/aft space) on all fitness variables were tested by an analysis of variance (ANOVA) for repeated measures. Differences between experimental conditions were tested with a posthoc Tukey test (paired comparisons). The main effects of experimental the conditions on each time difference variable (T0-T1, T0-T2, T0-T3, $\mathrm{T} 1-\mathrm{T} 2, \mathrm{~T} 1-\mathrm{T} 3$, and T2-T3) and the number of targets missed were tested multivariate with a MANOVA for repeated measures. Differences between experimental conditions were tested with a Hotelling $T^{2}$ test (paired comparisons). The selected level of significance in all tests was $p=.05$ (one-tailed).

\subsection{Results}

Postural discomfort in the buttocks-legs was significantly affected by the experimental conditions tested (Figure 11). As of the end of the fourth session (115-150 min after the start of the first session) discomfort for the fully confined condition was significantly higher than for the free condition. Furthermore, at the end of the fourth session (115 min after the start of the first session) discomfort for the fully confined $+15 \%$ for/aft space condition was significantly higher than for the free condition.

Postural discomfort in the neck-back was affected by the experimental conditions tested 


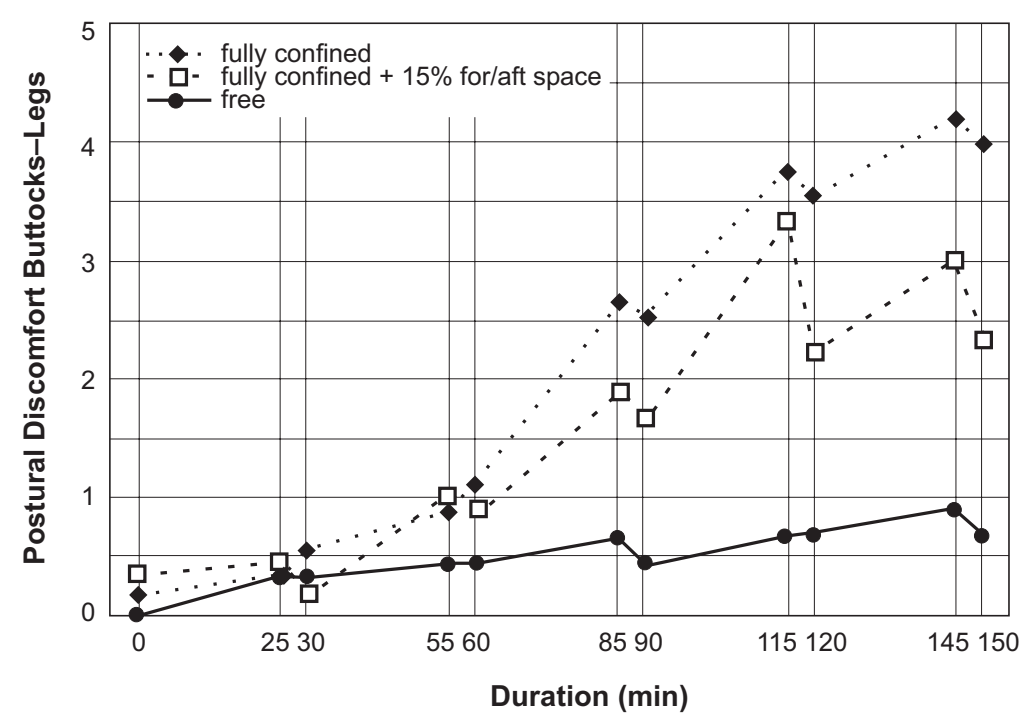

Figure 11. Experimental study in the laboratory: postural discomfort in the buttocks-legs in time versus experimental conditions (average group data).

$(p=.060)$. Postural discomfort for the fully confined condition was higher than for the free condition $(p=.055)$.

Eye discomfort was significantly affected by the experimental conditions tested average score. Average group scores, using a scale ranging from 0 (none) to 10 (extreme/maximal), were 0.87 for the free condition, 1.01 for the fully confined $+15 \%$ for/aft space condition, and 0.59 for the fully confined condition. Discomfort for the fully confined $+15 \%$ for/aft space condition was significantly higher than for the fully confined condition.

For physical fitness, mental fitness, postural discomfort in the right shoulder-arm, postural discomfort in the left shoulder-arm, headache, and observation performance no significant differences were found for each one of the pairs of experimental conditions. The highest average group score on headache, using a scale ranging from 0 (none) to 10 (extreme/maximal), was 0.67 .

\section{DISCUSSION AND CONCLUSIONS}

The two baseline studies provided insight into the effects of the duration of operation on the fitness and performance of crew members in a confined-space military vehicle. The average decrease in physical fitness of gunners $1-5$ in the simulator baseline study was $10.7 \%$ per hour. A similar value was found for the gunners in the field baseline study, i.e., $10.3 \%$ per hour. Postural discomfort for the gunners in both studies was located in the same body regions, i.e., neck, upper back, lower back, and buttocks. The average decrease in physical fitness of $10.9 \%$ for the commanders in the field baseline study was close to the results for the gunners, although discomfort was located in somewhat different body regions, i.e., lower back, buttocks, and upper legs (back side). Discomfort in the upper back and neck was absent for the commanders, but clearly present for the gunners. This is most likely due to the typical gunner posture (slight extension of the neck and slight forward inclination of the trunk) when using the optics for scanning the outside world and aiming. The technical performance of gunners $2-5$ in the simulator baseline study decreased by $4.1-4.4 \%$ per hour, while their observation performance in the field baseline study decreased by $29.8 \%$ per hour. As expected, it may be concluded that observation performance is more vulnerable for a decrease in fitness in time than technical performance.

The expectation that active breaks may have a positive effect on discomfort was confirmed in the field baseline study. With non-active breaks the fitness reduction rate for the commanders was $10.9 \%$ per hour, while the active breaks led to a 
significant reduction in the decrease of physical fitness, almost reducing it to zero. During nonactive breaks the reduction rate of mental fitness for the gunners was $12.6 \%$ per hour. Remarkably, there was a significant reduction in the decrease of fitness for the gunners when the commanders had active breaks, i.e., left their workstation. It looks like gunners only experience a break in task execution as a true break when their commander is not present.

No explanation was found as to why experimental conditions created differences in eye discomfort. The main hypothesis of the study (more confinement leads to lower fitness and performance) was only partially confirmed. The fully confined and (in a single case) fully confined $+15 \%$ for/aft space conditions created significantly lower fitness (more discomfort) than the free condition. Differences between the fully confined condition and the fully confined $+15 \%$ for/aft space condition could not be demonstrated. However, as illustrated by Figure 11, a tendency may be observed that a gradual increase in confinement is associated with a gradual increase in discomfort (fitness decline) per time unit. No differences among the experimental conditions were disclosed as regards performance. It might have been that discomfort was not high enough to affect performance like in the two baselines studies, or time to reach this higher discomfort level was too short. In this respect it is be mentioned that in the experimental workstation the trunk and neck were in the neutral posture, while posture in the two baseline studies was more unfavourable (a slight forward inclination of the trunk and a slight extension of the neck). Furthermore, in the two confined conditions the subjects had the opportunity to vary trunk posture (between neutral and somewhat forward inclined/twisted) due to the fact that a onehand operated control device was used instead of the two-hand operated control device of the Leopard 2 workstation used in the two baseline studies. Finally, it is considered good to know that no learning effect was found over the three days. A learning effect would have increased data variance, making it harder to disclose possible effects of the experimental conditions.
The gunners' and commanders' priorities for improvement of working conditions (Table 1) require an explanation. During discussions they clarified that the score for noise actually referred to complaints about the headset used for communication. The score for quality of seat pan and backrest referred to limited support during task execution. However, this is not so much a problem of the seat but of the position of the optics for scanning the outside world and aiming, leading to an unfavourable, slightly forward inclined, trunk posture. If we define high priority as an average score below 4.5 (halfway between rank 1, highest priority, and rank 8, lowest priority), the following items need to be addressed: quality of the seat pan and backrest (positions of optics), space available at the workstation (level of confinement), shocks and vibrations (possibly related to inadequate means for body support for the commander), and sustained seating and sustained task execution (duration aspects).

\section{REFERENCES}

1. International Organization for Standardization (ISO). Ergonomics-evaluation of static working postures (Standard No. ISO 11226:2000). Geneva, Switzerland: ISO; 2000.

2. European Committee for Standardization (CEN). Safety of machinery-human physical performance - part 4: evaluation of working postures and movements in relation to machinery (Standard No. EN 1005-4:2005). Brussels, Belgium: CEN; 2005.

3. Mathiassen SE, Christmansson M. Variation and autonomy. In: Delleman NJ, Haslegrave CM, Chaffin DB, editors. Working postures and movements-tools for evaluation and engineering. Boca Raton, FL, USA: CRC Press/London, UK: Taylor \& Francis; 2004. p. 330-66.

4. Drury CG, Paquet VL. Performance. In: Delleman NJ, Haslegrave CM, Chaffin DB, editors. Working postures and movements-tools for evaluation and engineering. Boca Raton, FL, USA: CRC Press/London, UK: Taylor \& Francis; 2004. p. 403-23. 
5. Corlett EN, Bishop RB. A technique for assessing postural discomfort. Ergonomics. 1976;19:175-82.

6. Borg G. A category scale with ratio properties for intermodal and interindividual comparisons. In: Geissler HG, Petzold P, editors. Psychophysical judgement and the process of perception. Amsterdam, The Netherlands: North-Holland; 1982. p. 25-34.
7. Van der Grinten MP, Smitt P. Development of a practical method for measuring body part discomfort. In: Kumar S, editor. Advances in industrial ergonomics and safety IV, Proceedings of the Annual International Ergonomics and Safety Conference, Denver, CO, USA. London, UK: Taylor \& Francis; 1992. p. 311-8. 